\title{
The Significance of Enterprise Resource Planning (ERP) Systems in Aviation Industry
}

\author{
Sherzad Ramadhan, PhD Candidate \\ Hassan Koutaini, PhD Candidate
}

Bucharest Academy of Economics Studies (ASE), Romania

Ass. Lec. Mr. Karwan Ali Ali

University of Zakho, Iraq

Doi:10.19044/esj.2019.v15n10p100 URL:http://dx.doi.org/10.19044/esj.2019.v15n10p100

\begin{abstract}
Rapid globalization has introduced several problems in the business environment. These problems have added complexities in decision-making and intensified competition. As a result, organizations are forced to come up with comprehensive ways of mitigating the challenges. That together with the ever-changing needs of the airlines' customers have heightened the value attached to information systems as a way of solving problems and gaining competitive advantage. Enterprise Resource Planning (ERP) system is one of the most popular systems in many organizations. However, some organizations have reported negative feedback pertaining its value. In this view, the primary objective of this study is to discern the significance of ERPs in the aviation sector. The study will accomplish that goal by focusing the research on Kenya Civil Aviation Authority, Delta Airlines, United Airlines, and the aviation sector of the Kingdom of Saudi Arabia. These cases were selected because they have successfully implemented ERP and experienced several challenges before they successfully implemented the system. The study employed secondary research methods to collect data. Literature affiliated to the topic of study particularly materials that focused on the selected cases were utilized. Critical analysis was conducted and the findings presented. To increase validity and reliability, Nvivo software was used whereby interpretive and intuitive methods were utilized. The software method gave similar results as those obtained through manual methods.
\end{abstract}

Keywords: ERP, Significance, Aviation industry, success, Implementation. 


\subsection{Introduction}

\subsection{Background of the Study}

In the past three decades, a plethora of information systems have been adopted at a rate consistent with the Moore's Law of increasing the computing power for every eighteen months (Laudon, 2010). According to data provided by the US Department of Commerce, the amount of funds committed to information technology, specifically hardware and software, by the private sector increased from 32\% of all invested capital in 1980 to $52 \%$ in 2007. Other countries have followed suit as different industries and firms continue to establish new business objectives as they work towards the realization of their respective corporate goals. To achieve organizational objectives, it is important that industries work to ensure that they align information technology to the organizational mission objectives (Ayeni, Ball, \& Baines, 2016). Despite the vast resources needed for their implementation, enterprise resource planning has significant benefits that include increased productivity, better positioning of the organization in the long-term, and expansion of the revenue base. In the aviation industry, these benefits allow for a reduction in the cost of the fares hence better customer experience (Khan \& Faisal, 2015). As the aviation industry grows in the exponential rate, ERP seeks to help organizations when it comes to integrating the integration of the manufacturing management, discrete production, human -capital management, electronic data interchange, entire supply chain and improvement in the overall quality management (Flouris \& Oswald, 2016). While these systems have been adopted at an increasingly fast rate, very little attention has been directed to the factors that drive or put off the utilization of the systems in the aviation sector. Thus, this paper entails the benefits of ERP that attract airlines as well as the challenges associated with ERP that discourage airlines to adopt the systems.

\subsection{Problem statement}

Both big and small businesses are vital to an economy. Just like small firms, big multinationals like Delta Airlines and Emirates encounter many challenges when installing technologies like ERP. While ERP systems were initially a reserve for big companies, Muscatello et al. (2009) point out that small and medium companies have in the recent past become increasingly interested in the systems. The same trend has been noted in the aviation sector where big airlines and manufacturing companies led the way for small airlines and manufacturers. That raises questions as to what factors motivate them to implement the technology.

Many studies on ERPs have focused on fields outside the aviation sector. The studies underscore the significance of employee training as a prerequisite for successful ERP implementation. In particular, Muscatello et 
al. (2009) point out that employee training is a key factor for the success of ERP, especially after the implementation stage. However, the scholars do not indicate the effects of quality training together with age, gender, and frequency of use on the perception and attitudes towards the technology. Another study by Aleqtisadih (2010) revealed that the size of an organization, the rate of employee turnover, the age of the organization, and location affected the adoption of ERP. Despite these findings, studies that focused on the aviation industry were noted to have scant information.

The few studies relating to ERP in the aviation industry focused on the aviation industry of Saudi Arabia. Saudi Arabia is one of the countries with the highest rate of ICT failure and incurs huge losses. Aleqtisadih (2010) noted that Saudi Arabia incurred more than \$0.5 trillion losses in 2010 due to ICT failure. Regardless of the losses, investments in technological advancements have been on the rise. The increasing figures on investments in IT are one of the motivations to examine the driving factors for the adoption of ERP systems in that region particularly the Emirates Airline. Therefore, the study will use Delta Airlines, Emirates, United Airlines, Boeing Company, Airbus Company, and Kenya Civil Aviation Authority as case studies to examine these issues.

\subsection{Research Questions}

1. How are ERPs used in the aviation industry?

2. How has the technology changed (benefits) in the aviation sector?

3. What factors motivate airlines and the aviation sector at large to implement ERPs?

4. What are the challenges encountered during implementation and after implementations?

\subsection{Implications of the Study}

The objective of the study is to examine the motivations behind the adoption of enterprise resource planning system in the aviation sector. The study will use Delta Airlines, Emirates, United Airlines, Boeing, Airbus, and Kenya Civil Aviation Authority as case studies.

This study will provide rich knowledge on the use of management information systems and the impact of user level of training and attitude on efficiency. According to Avison and Fitzgerald (2008), the causes of failures of information systems have been majorly human factors as opposed to technical factors. Therefore, the findings of this study will help shed light on the significance of attitudes and level of knowledge of the user on the efficiency of the organization and possibly its likelihood of adoption of future systems. In addition, the findings will demonstrate how the ERPs have 
changed the aviation sector and enabled major airlines and civil authorities to benefit. Importantly, the findings will provide insights into the challenges and limitations of ERPs as well as the likely solutions.

\subsection{Literature Review}

Literature review entails a critical analysis of the scholarly literature related to the topic of study. Thus, this section presents results of critical analysis of views and opinions of different scholars on the drivers behind the adoption of ERPs in the aviation sector. The section also presents the benefits and challenges associated with the utilization of ERPs.

\subsection{Theoretical Framework}

Numerous theories have been put forward to explain the relationship between motivations and adoption of technology. In this study, two theories are used to help achieve the study goals. The two theories include the Technology Acceptance Model (TAM) and the Diffusion of Innovation Theory (DOI).

\subsubsection{Technology Acceptance Model}

TAM was first coined by Davis (1986) and is anchored on people's attitudes and behavioral theories. According to the model, the level of acceptability of a system is determined by perceptions on the ease of use and perceptions on its usefulness. Perceived usefulness relates to the extent at which the user thinks the system will enhance his performance while ease of use relates to the extent to which the user thinks the use of the system will be effortless (Usolo \& Shoyelu, 2010). Given that the features and complexity of an information system influence the perceived usefulness and perceived ease of use, a system with little complexity will have high perceived usefulness and high perceived ease of use. Thus, the ability of this model to estimate the acceptability of an information system with respect to perceived usefulness and perceived ease of use will significantly help in answering the research questions.

\subsection{Situational Analysis of Adoption of Enterprise Resource Planning (ERP) System in Business Organizations and the Aviation Sector}

Enterprise Resource Management is understood by many as a business system that integrates a wide array of software which centralize business functions. Amidst increase in competition, businesses have leaned towards adoption of the latest technology innovation to gain competitive advantage. As a result, the significance and value of ERP system has increased in the recent years hence its adoption has become essential if not a key part of the business management. Besides its advantages, ERP has been characterized as 
an expensive and extremely complex system to use (Kiarie \& Wanyama, 2017). Previous users and business managers point out that ERP requires intensive planning and careful execution. That could explain why many organizations have attempted to exploit the benefits of the technology but instead incurred heavy losses in terms of time and money. The losses are more in the developing countries because the organizations do not have access to well-developed ICT infrastructure as well as inadequate investments in research and development. However, evidence suggests that organizations that effectively implement ERP systems enjoy unlimited advantages (Kiarie \& Wanyama, 2017).

Since its development in the early 1990s by the Gartner Group of Stamford, ERP has undergone several stages of evolution. It is important to understand that ERP was an evolution from the Material Requirement Planning (MRP)in the 1960s, MRP II in 1980s and finally to ERP the 1990s. MRP II was originally created to help manufacturers in planning through the use of computer tools (Kiarie \& wAnyama, 2017). Further improvements on ERP resulted in the integration of the whole supply chain which made easy for information sharing. In 2006, more improvements were done which expanded the scope of ERP to include tools for e-commerce as well as address issues related to customer relationship management and management of the supply chain (Kiarie \& Wanyama, 2017).

\subsection{Drivers for the Adoption of ERP Systems in Organizations}

According to Hammer (2009), both internal factors and external factors motivate organizations to adopt ERPs. The internal factors relate to the organizational culture and the available resources. Organizational culture not only affects the workplace interactions between the employees but also influences their attitudes change which is a key aspect in the adoption and implementation of ERPs. On the other hand, Sawah et al. (2008) points out that the need to improve performance, better access to information, cut costs, and increase efficiency are some of the drivers for the adoptions of ERPs.

According to Bingi et al. (2009), training "refers to learning experiences designed to enhance the shortterm and/or long-term job performance of individual employees". In this respect, training is viewed as part of an on-going developmental process. Training needs to be linked with the organizational mission. So, when governments plan their training activities, they need to provide the link with the organizational mission and local budget and implementation. Considerate the occurrence of employee training needs understanding of all the modifications that take place as a result of learning. As the producer of new skills, workers' training is located inside a broader planned background of human resources management, i.e. worldwide managerial organization, as a designed staff education and 
development, both individual and group, with the goal to benefit both the organization and employees. According to Bingi et. al, (2009) in his book Personnel and Human Resource Management, training is a movement intended for gaining of accurate knowledge and skills for the purpose of an occupation or chore.

In addition, increased regulation and control has forced many organizations around the world to adopt ERP systems (Ideal Engineers, 2014). In the same way, Nzuki and Musyimi (2013) point out that the need to have a centralized source of information, improve processes, enhance customer satisfaction, and improve decision-making are some of the motivating factors for organizations to adopt ERP.

\subsection{Benefits of ERP Systems in Organizations and in the Aviation Sector}

While there are several reasons that drive organizations to adopt ERP systems, Laukkanen, Sarpola, and Hallikainen (2007) summarize them to save on costs, enhance quality, enhance the activities of the human resource management, and increase productivity. Increase in globalization has made business needs to become more dynamic and complex hence the demand for implementation of innovative information systems. ERP systems meets that need by providing top executive team with a centralized real-time snippet of the progress of business activities. In addition, ERP systems streamline the workflow especially where there is automation of activities. For instance, the system avails information and paperwork to operational managers and senior managers for approval at the right time (Almajali, Ma'sadeh and Tarhini, 2016).

In addition, ERP systems ensure there is no duplication of efforts by availing key information from different departments in a central point of access hence enabling leaders to analyze the business from different perspectives. However, this puts more weight or impact on the user's adoption of the system. Nonetheless, Wickramasinghe and Karunasekara (2012) show that the single point of view allows managers to access the performance of the junior employees and analyze that data at granular level in real-time. As a result, the manager has more control and better access to information leads to increase in productivity.

\subsection{Challenges Facing the Adoption of ERP}

The vast majority of literature on this topic indicate that lack of skills is the primary challenge facing organizations in the adoption and implementation of ERP systems (Summer, 2000; Avison \& Fitzgerald, 2008). The scholars posit that absence of adequate knowledge and internal expertise coupled with poor combination of external and internal expertise exacerbates the problem of retaining IT experts. Given that IT experts are critical to train 
the staff that will be using the ERP system, attrition results in poor training which in turn leads to difficulties in using the technology.

In addition, the Enterprise Resource Planning system is expensive. The high buying price is heightened by costs for adjustments since the system is not tailor made to the customer's needs. Further, some organizations lack IT experts and have to hire external labor for installation and testing. Also, Maheshwari, Kumar, and Kumar (2010) established that many organizations encounter difficulties in getting their employees to highlight areas that need modification in the early stages of implementation.

Sahin (2006) noted that compatibility with other systems is a major challenge in the implementation of ERPs. Since business organizations have other systems installed, there is the need to realign them with ERP which means making technological and organizational changes. Even after the changes, the integrated nature of enterprise resource planning system causes major alterations on the organizational structure, the workflow, and way in which employees execute their responsibilities. That issue was observed by Sawah, Fattah, Mohamed and Rasmy (2008); Kerr and Houghton (2014) to necessitate Business Proper Reengineering (BPR) and a change in the organizational culture all of which are extremely difficult hence majority of the employees are resistant to the processes/changes. Due to these problems, many organizations give more attention to consultancy and tools (hardware and software) and underestimate the importance of employee participation in the process of implementation. The failure to include users and train them during implementation results in negative attitudes and perceptions toward the ERPs which in turn leads to decline in efficiency, contrary to its objective of enhancing efficiency and productivity (Kerr \& Houghton, 2014).

\subsection{Research Methodology}

The study adopted a secondary research approach. According to McQuarrie (2015), secondary research method entails a review and synthesis of existing data and materials collected by other researchers through primary research methods. Secondary research is also referred to as desk research as it entails meta-analysis of statistics presented through publications and reports (McQuarrie, 2015). In this study, the researcher examined research presented by other authors regarding the methods used in the design of offshore floating wind turbines and developed a critical analysis of the research. According to McQuarrie (2015), secondary research method has several advantages:

1.It is fast and cheap as the information is readily available.

2.It acts as a guide to any subsequent research after the secondary research

3. Sometimes, it is the only available source of information for specific organizations such as the government. 


\section{Disadvantages}

1.The literature presented lacks specificity and does not address the question at hand.

2.Some secondary data can be of low quality and outdated (McQuarrie (2015).

3.Secondary data like customer databases can only describe the existing customers.

The researcher collected information from journals and presentations from various conference proceedings. Internet was used to get access to these publications and journals. In particular, the researcher used materials that focused on the significance of ERPs in the aviation sector. The researcher emphasized on those sources that focused on the Kenya Civil Aviation Authority, Saudi Arabian Aviation sector, and the United States Aviation sector. The focus on these sources was strategic in a manner that it enabled discover some of the key factors that drive the adoption of ERPs, ingredients for its successful implementation, and the value added after implementation.

In order to ensure reliability and validity of the findings obtained using this method, several sources must be used. Therefore, the study utilized as many sources as possible in order to obtain a wide range of facts on the subject. From the broad spectrum of findings, the researcher used Nvivo software to establish the relationships and map out the final findings. Also, intuitive and interpretive methods of analysis were used to complement the software method. After the comparison, the final report was written as detailed in the next section.

The decision to combine both secondary research method (manual method) and the software method where the Nvivo software was used came from the understanding that sometimes researchers fail to identify relationships and key themes in research publications due to mere human mistakes. Thus, the software method eliminates this problem since it is unbiased and not prone to human mistakes. As a result, all the key themes and relationships between different publications and the subject topics are clearly mapped out without bias. In addition, the software method is faster and cost saving hence feasible to adopt as an additional method for verification, comparison, and validation of the results. After the comparison, the final report was written as detailed in the next section.

\subsection{Findings}

One of the fundamental result obtained from the usage of ERP within the aviation industry is that it enhances a higher level of performance within the organization. In today's world, it is becoming harder to cope with competition in organizations. Organizations are targeting to stay more efficient. Also, enhance customer services, guarantee supervisory compliance, 
and more efficiently use resources to remain competitive in the market. However, enterprise resource planning (ERP) frameworks are good news (Müller, et, al., 2014). These systems play an essential part when it comes to organizing and processing financial information. Additionally, it enhances the general business expansion functions in the long-term and short -term goal. Adoption of ERP frameworks in an aviation company can also improve the efficiency of the procedures. Minimize costs, restructuring processes, and increase productivity.

The analysis of the results from the findings shows that the use of ERP strictures will give a sole and a basis of truth and allows the company to enhance communication amongst departments (Müller, Wittmer \& Drax, 2014). ERP systems prevent keeping vital spreadsheet and documents on individual computers of employees. They promote storing significant documentation in a centralized database to eradicate redundancies. Every time a piece of data gets in the company's ERP framework, it inevitably moves to every region that may require or utilize that entered in the ERP framework. It will enhance information accuracy since there no requirement for duplicate entry of any portion of data (Müller, Wittmer \& Drax, 2014). A company can operate smoothly and misuse no time searching down for data and authenticating its accuracy. Such can be made possible when the company's employees can access the specific data they require swiftly, all from a similar integrated framework. With all the data readily available, the company can examine and regulate its production matters as it comes up with positive strategies towards the realization of its key operational goals within the aviation industry. Rather than waiting for hours and months to attend to issues and make an adjustment.

ERP frameworks help eliminate departmental blind sports within the aviation industry. Departments within the aviation industry function best when autonomous is encouraged. However autonomous can build the issue of blind spots (Müller, Wittmer \& Drax, 2014). Lack of insights into other sectors, the company can misuse resources duplicating efforts to resolve the same matter. Central portal aids department customize information, share information, and make the individual report. Besides, it endorses collaboration and sharing of resources and knowledge (Oke \& Onyegiri, 2017). Due to cloud technology, there is a new generation of collaboration. ERP equipment has taken advantage of such techniques and integrating results that can even control work association amid remote regions in real-time. It endorses an excellent interdepartmental conveyance and resource allotment. In the end, company departments are creating stronger relationships and associating in more projects.

Further research from the research methodology shows that ERP offers customer service and sales with proper communication channels, which results 
in sale predicting and excellent customer care. In suppliers and customers, an ERP framework enhances both the company's front-end and back end connection through building an efficient transporting and conveyance structure. It restructures transportation time, producing a good experience for the customers (Kumar, Sharma \& Agarwal, 2015). The supplies are very satisfied since they receive reliable customers about the type of service that people receives in the aviation sector. In the end, the industry in aviation can align the amount with the demand. ERP structures involve customer prioritization. Sales data that gathers in a given time offers an opportunity to enhance the client's retention. Additionally, based on the nature of service about the ERP, the customer will feel that they are satisfied as the aviation industry work to reduce the possible challenges that are associated with the department. Notably, the increase in the growth and competition in different industries such as the aviation, it is highly essential especially for the airliner to come up with better ways and mechanisms that they can use about adopting the ERP to help streamline their operations while at the same time reducing the overall operational cost.

\subsection{Conclusion and Recommendations \\ 5.1 Conclusion}

Enterprise Resource Planning systems are used to accomplish critical tasks not only in the aviation sector but also in other sectors. The main objective for the implementation of ERP is to enhance the realization of the organizational goals as well as to offer integrated IT solutions. In addition, ERPs help in the identification of opportunities by eliminating the duplication of efforts. Importantly, ERP helps to streamline communication between departments which results in better customer service and better service delivery. The aviation industry is a service-oriented sector and the quality of services offered together with the level of customer service have huge impact on the overall performance of the company. Therefore, the adoption of ERPs by airlines helps to improve the overall performance.

The numerous benefits of ERP are only enjoyed after successful implementation. As indicated in the discussion above, several factors could affect the realization of this end-goal. Such factors include the availability of resources, organizational support, and organizational culture. Therefore, the usefulness of the ERP within an organization depends on the management's ability to facilitate system service and provide financial aspects of the system. It is also important that the organization through its members adopt a positive culture that will promote adaptability of the ERP within the organization by enhancing open environmental conditions. 


\subsection{Recommendations}

As noted in the discussion above, training is vital for the success of ERP in an organization. Through training, users learn to better strategies that they can use the system as they seek to enhance organizational success (Azevedo, Romão \& Rebelo, 2014). Many studies have examined the influence of organizational support and availability of resources on the success of ERP. In addition, literature used in this research entailed the connection between alignment of organizational policies with the ERP properties. However, scant information is available on how to align the organizational culture and policies in a manner that creates a conducive environment for the implementation of ERP. Therefore, future studies should concentrate on the ways in which organizations, not only in the aviation industry but also in other sectors, can sketch and develop an environment that encourages employee training and offers support to the learning employees during implementation of ERPs. As noted in the theoretical framework, perceived usefulness is based on the ease of use of the system. Given that many ERP systems are not customized, future studies should explore how organizational culture can help present ERPs as easy to use and valuable to the organizations.

\section{References:}

1. Aleqtisadih, n. (2010, Feb 11). The world in the use of communications technology and the absence of an Arab. Retrieved from ReutersSweden, www.aleqt.com/2010/02/11/article_348191.html

2. Almajali, D. A., Masa'deh, R. E., \& Tarhini, A. (2016). Antecedents of ERP systems implementation success: a study on Jordanian healthcare sector. Journal of Enterprise Information Management, 29(4), 549-565.

3. Avison, D. \& Fitzgerald, G., (2008) Information systems development (2008) 4 th Edition. McGraw-Hill Education

4. Ayeni, P., Ball, P., \& Baines, T. (2016). Towards the strategic adoption of Lean in aviation Maintenance Repair and Overhaul (MRO) industry: An empirical study into the industry's Lean status. Journal of Manufacturing Technology Management, 27(1), 38-61. doi:10.1108/jmtm-04-2015-0025

5. Azevedo, P. S., Romão, M., \& Rebelo, E. (2014). Success factors for using ERP (Enterprise Resource Planning) systems to improve competitiveness in the hospitality industry. Tourism \& Management Studies, 10, 165-168. Retrieved from http://www.tmstudies.net/index.php/ectms/article/view/617

6. Bingi, P., Sharma, M. K., \& Godla, J. K. (1999). Critical issues affecting an ERP implementation. IS Management, 16(3), 7-14. 
7. Davis, F. D. (1989). Perceived usefulness, perceived ease of use, and user acceptance of information technology. MIS quarterly, 319-340.

8. Flouris, T. G., \& Oswald, S. L. (2016). Designing and executing strategy in aviation management. Routledge. doi.org/10.4324/9781315576718

9. Ideal Engineers (2014) A press release dated [Johannesburg, 22 September 2014] retrieved March, 17 2019, from www.itweb.co.za/

10. Kerr, D., \& Houghton, L. (2014). The dark side of ERP implementations: narratives of domination, confusion and disruptive ambiguity. Prometheus, 32(3), 281-295.

11. Kiarie, J., \& Wanyama, M. W. (2017). Factors Influencing The Adoption And Implementation Of Enterprise Resource Planning (Erp) System In The Smes Sector. Journal of Business and Strategic Management, 2(1), 62-85.

12. Kumar BR, R., Sharma, M. K., \& Agarwal, A. (2015). An experimental investigation of lean management in aviation. Journal of Manufacturing Technology Management, 26(2), 231-260. Doi: 10.1108/jmtm-12-2013-0174

13. Laudon, K.C and Laudon, J.P (2010) Management information systems: Managing the digital firm 11th Edition Page 196 \& 35 Upper Saddle River, New Jersey: Pearson Prentice Hall (Pearson Education Inc.).

14. Laukannen, S., Sarpola, S., \&Hallikainen, P., (2007) Enterprise size matters: Objectives and constraints of ERP adoption. Journal of Enterprise Information Management 20 (3) 319-344

15. Maheshwari, B., Kumar, V. \& Kumar, U. (2010) Delineating the ERP institutionalization process: go live to effectiveness. Business Process Management Journal, (16)4 774-771

16. McQuarrie, E. F. (2015). The Market Research Toolbox: A Concise Guide for Beginners. Thousand Oaks : SAGE Publications, Inc.

17. Müller, R., Wittmer, A., \& Drax, C. (2014). Aviation risk and safety management. Cham: Springer, 45-48.

18. Muscatello, J.R., Small, M.H. \& Chen, I.J. (2009). Implementing ERP in small and midsize manufacturing firms. International Journal of Operations \& Production Management, Vol. 23, 850-71.

19. Nzuki, D., \& Musyimi, D. (2015). Adoption of Enterprise Resource Planning Systems in Kenya : A Case of Selected Manufacturing Firms in Nairobi Metropolitan.

20. Oke, S. A., \& Onyegiri, I. E. (2017). A grey relational analytical approach to safety performance assessment in the aviation industry of a developing country. Engineering and Applied Science Research, 44(1), 1-15. doi:10.1504/ijbis.2015.070205 
21. Sahin, I. (2006). Detailed review of Rogers' diffusion of innovations theory and educational technology-related studies based on Rogers' theory. Turkish Online Journal of Educational TechnologyTOJET, 5(2), 14-23.

22. Sawah, S. E., Fattah, A., A., E., Mohamed, T. \& Rasmy, H. (2008) A quantitative model to predict the Egyptian ERP implementation success index. Business Process Management Journal, 14(3) 288 306

23. Sumner, M., (2000) Risk factors in enterprise wide/ERP projects: Journal of Information Technology. 15, 317-327.

24. Usoro, A. and Shoyelu, S. (2010) Task-Technology Fit and Technology Acceptance Models Applicability to e-Tourism. Journal of Economic Development, Management, IT, Finance and Marketing, 2(1) 1-32

25. Wickmarasinghe, V., and Karunasekara, M., (2012). Behavior \& Information Technology. Online Journal of Education Technology. 31(9) 873-887 